\title{
Long-lived binary tunneling spectrum in the quantum Hall Tomonaga-Luttinger liquid
}

\author{
K. Washio, ${ }^{1}$ R. Nakazawa, ${ }^{1}$ M. Hashisaka, ${ }^{1}$ K. Muraki,${ }^{2}$ Y. Tokura,${ }^{2,3,4}$ and T. Fujisawa ${ }^{1, *}$ \\ ${ }^{1}$ Department of Physics, Tokyo Institute of Technology, 2-12-1 Ookayama, Meguro, Tokyo, 152-8551, Japan \\ ${ }^{2}$ NTT Basic Research Laboratories, NTT Corporation, 3-1 Morinosato-Wakamiya, Atsugi, 243-0198, Japan \\ ${ }^{3}$ Graduate School of Pure and Applied Sciences, University of Tsukuba, Tsukuba, 305-8571, Japan \\ ${ }^{4}$ International Education and Research Center of Science, Tokyo Institute of Technology, 2-12-1 Ookayama, Meguro, Tokyo, 152-8551, Japan
}

(Received 19 January 2015; revised manuscript received 11 January 2016; published 5 February 2016)

\begin{abstract}
The existence of long-lived nonequilibrium states without showing thermalization, which has previously been demonstrated in time evolution of ultracold atoms, suggests the possibility of their spatial analog in transport behavior of interacting electrons in solid-state systems. Here we report long-lived nonequilibrium states in one-dimensional edge channels in the integer quantum Hall regime. An indirect heating scheme in a counterpropagating configuration is employed to generate a nontrivial binary spectrum consisting of high- and low-temperature components. This unusual spectrum is sustained even after traveling 5-10 $\mu \mathrm{m}$, much longer than the length for electronic relaxation (about $0.1 \mu \mathrm{m}$ ), without showing significant thermalization. This observation is consistent with the integrable model of Tomonaga-Luttinger liquid. The long-lived spectrum implies that the system is well described by noninteracting plasmons, which are attractive for carrying information for a long distance.
\end{abstract}

DOI: 10.1103/PhysRevB.93.075304

\section{INTRODUCTION}

Dynamics of quantum many-body systems often result in thermalized states characterized, for example, by the Fermi distribution function [1]. Exceptional cases have been discussed for integrable systems, where an isolated system may be left in a nonequilibrium steady state [2]. This intriguing aspect has been studied in isolated systems like one-dimensional (1D) cold atomic chains, where temporal evolution of the system can be measured after a sudden change of the interaction (quantum quench) $[3,4]$. Complementary experiments in transport measurement would allow us to study spatial evolution of electronic states traveling from a noninteraction region to an interacting region (spatial analog of quantum quench). The Tomonaga-Luttinger (TL) liquid, which has been identified in various $1 \mathrm{D}$ wires and quantum-Hall edge channels [5-9], is known as an example of integrable systems. Theoretically the Coulomb interaction between electrons is absorbed under bozonization into noninteracting plasmon modes of collective density excitations [10-12]. Therefore, an ideal TL liquid never thermalizes as plasmon excitations are conserved during the transport [13-15]. Quantum-Hall edge channels are suitable for demonstrating this nonthermalizing behavior, as they are well isolated from the environment. Actually, coupling to the phonon bath is sufficiently weak [16], and backscattering that is unwanted for the TL model is highly forbidden [17,18]. Moreover, tunneling spectroscopy with a quantum dot (QD) allows us to investigate spatial evolution of the electronic spectrum in a tailored geometry [19]. Previous experiments have identified electronic relaxation from singleparticle excitations to collective excitations (plasmons) in terms of plasmon boundary scattering known as spin-charge separation [20-23] and fractionalization [24,25] in the TL physics. However, the absence of thermalization as a hallmark of an integrable system has not been addressed, as the resulting states were always close to thermalized states showing a trivial Fermi distribution [20,26,27].

*Email: fujisawa@phys.titech.ac.jp
In this paper we show that quantum-Hall edge channels can actually support long-lived nonequilibrium states. For this purpose, nontrivial binary spectrum composed of hot and cold carriers is prepared by an indirect heating scheme using weakly coupled counterpropagating edge channels. Quantum dot (QD) spectroscopy clearly reveals that the carriers with the nontrivial binary spectrum propagate over a long distance, much longer than the length required for electronic relaxation, without thermalization into a trivial Fermi distribution. This nonthermalizing characteristic is consistent with the TL model and encourages us to study nonequilibrium coherent plasmon transport in the system.

The paper is organized in the following way. After describing an artificial TL liquid formed in quantum-Hall edge channels (Sec. II A), we propose an excitation scheme to obtain nontrivial binary spectrum (Sec. II B). The binary spectrum is obtained from experiments and simulations. For experiments, we describe the device structure and measurement scheme (Sec. III A), and compare energy spectra in two regions in distinct geometries (Secs. III B, III C, and III D) by considering the heat flow in the system (Sec. III E). The binary spectrum is found in one of the regions, where small amplitudes of highfrequency plasmons are excited. For simulations, we derive plasmon eigenmodes of the system (Sec. IV A), and show that an approximate binary spectrum appears as a nonequilibrium steady state in the quantum-quench simulations (Sec. IV B). Finally, we discuss the coupling strength ( $\mathrm{Sec} V \mathrm{~A}$ ), the similarities and differences between the experiments and simulations (Sec. V B), some conserved quantities during the transport (Sec. V C), and possible relaxation mechanisms in the quantum-Hall system (Sec. VD) before summarizing the paper.

\section{ARTIFICIAL TL LIQUID}

\section{A. Plasmon excitation in edge channels}

Figure 1(a) illustrates the interacting quantum-Hall edge channels we study, where right $(\eta=\mathrm{r})$ and left $(\eta=\ell)$ moving electronic channels with spin $\sigma \in\{\uparrow, \downarrow\}$, labeled $(\eta, \sigma)$, are 
(a)
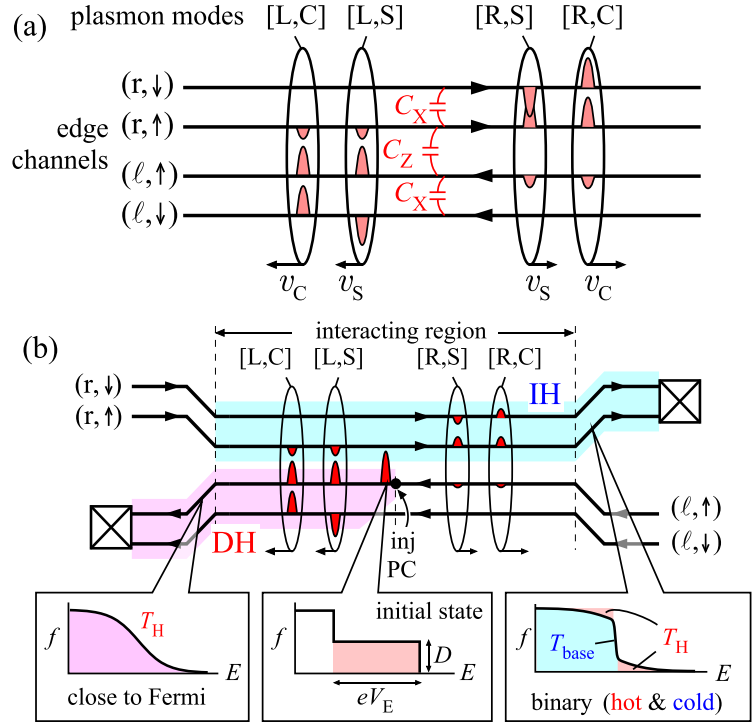

FIG. 1. (a) Schematic diagram of interacting quantum Hall edge channels $(\mathrm{r} / \ell, \uparrow / \downarrow)$ with nearest neighbor coupling capacitances $C_{\mathrm{X}}$ and $C_{\mathrm{Z}}$. Charge wave packets for plasmon eigenmodes [L/R,C/S] are illustrated. (b) The indirect heating scheme for interacting quantum-Hall edge channels $(\mathrm{r} / \ell, \uparrow / \downarrow)$. Each time a single-electron charge wave packet is injected from a PC to $(\ell, \uparrow)$ as shown by "inj," four plasmon charge wave packets in the eigenmodes $[\mathrm{L} / \mathrm{R}, \mathrm{C} / \mathrm{S}]$ are generated. Carriers are drained to ohmic contacts marked by $\otimes$. The energy distribution function $f(E)$ shown at the bottom insets exhibits a double step structure for the initial state (the central inset), close to Fermi distribution at $T_{\mathrm{H}}$ in the $\mathrm{DH}$ region (the left inset), and an unusual binary spectrum with two temperatures $T_{\mathrm{H}}$ and $T_{\text {base }}$ in the $\mathrm{IH}$ region (the right inset).

interacting. Such a geometry can be prepared by depleting a narrow central region of a two-dimensional electron system (2DES) in a magnetic field at the filling factor $v=2$ [24]. In the absence of tunneling and the presence of electrostatic interaction between the channels, the system mimics an ideal 1D wire described by the standard spin-full TL model [28-31]. The Coulomb interaction yields four plasmon eigenmodes, labeled $[d, m]$, i.e., left $(d=L)$ and right $(d=R)$ moving charge $(m=C)$ and spin $(m=S)$ modes, as will be derived in Sec. IV A. The charge and spin modes have symmetric and antisymmetric charge distributions, respectively, in the primary channels (spin-charge separation), where the charge velocity $v_{\mathrm{C}}$ is generally greater than the spin velocity $v_{\mathrm{S}}$. The interaction between counterpropagating channels generates small amount of dragged charges reverse-travelling in the subsidiary channels (charge fractionalization), as illustrated by coupled wave packets in Fig. 1(a) [24,32,33].

In a practical device, such interacting channels can be formed in a finite region, outside of which the left and right moving channels are spatially separated as shown in Fig. 1(b). Suppose that a nonequilibrium charge is injected into one of the channels, $(\ell, \uparrow)$ [illustrated as an arrow marked by "inj”'] from a quantum point contact (PC). The initial electronic state in $(\ell, \uparrow)$ exhibits a double-step profile in the energy distribution function $f(E)$, where the width and height are given by the excitation energy $e V_{\mathrm{E}}$ and the tunneling probability $D$, respectively, of the $\mathrm{PC}$, as shown in the central inset at the bottom of Fig. 1(b). However, the interaction alters the electronic spectrum significantly during the transport [20].

In the plasmon picture, charge wave packets randomly injected from the $\mathrm{PC}$ can propagate in a noninteracting manner. Each time a single electron is injected into $(\ell, \uparrow)$, an initial wave packet, shown just on the left side of the injection point, is generated. It can be described as a linear superposition of the four plasmon eigenmodes. Since the charge and spin modes have different velocities, the injected charge splits into four plasmon packets as illustrated. We will refer to this splitting as electronic relaxation in the sense that a single-particle excitation in one channel is relaxed into collective charge excitations over the channels. This takes place within a length $l_{\mathrm{el}}=2 \hbar v_{\mathrm{C}} v_{\mathrm{S}} / e\left(v_{\mathrm{C}}-v_{\mathrm{S}}\right) V_{\mathrm{E}}$, which is approximately $100 \mathrm{~nm}$ at the excitation voltage $V_{\mathrm{E}}$ of $1 \mathrm{mV}$ for typical velocities $v_{\mathrm{C}}=$ $2-5 \times 10^{5} \mathrm{~m} / \mathrm{s}$ and $v_{\mathrm{S}}=2-5 \times 10^{4} \mathrm{~m} / \mathrm{s}$ at $v=2[21,34,35]$, from the injection point. When random charge packets are successively injected from a biased PC, channels are filled with incoherent ensemble of plasmon wave packets. A fast wave packet in the charge mode can catch up with a slow packet in the spin mode, and overtake it without any scattering as the plasmons are noninteracting. Therefore, the excitation of noninteracting plasmons cannot be thermalized [13,36,37].

\section{B. Nontrivial binary spectrum}

We next show that nontrivial binary spectrum can be generated in the excitation scheme described in the previous subsection. To see this, it is important to note that electron-hole excitations (and thus the energy spectra) in all channels are correlated with each other as they all originate from the common injection process at the PC. Most of the generated wave packets flow to the downstream of the injection point, which we refer to as "direct-heating (DH) region" [the red region in Fig. 1(b)]. Previous studies have shown that the spectrum in this region can be described by an approximate Fermi distribution at temperature $T_{\mathrm{H}}$, as schematically shown in the left inset [20]. This might not contradict the nonthermalizing nature of TL model, as the theory has shown that the electronic relaxation results in an approximate Fermi distribution for this case [26,27]. However, the experiment on the DH region was not successful in identifying the presence of long-lived nonequilibrium states.

Here, we focus on the spectrum in the counterpropagating channels, which we refer to as "indirect-heating (IH) region" (the blue region). Because of the small amplitude of the correlated excitations, the spectrum in the IH region should have a small fraction of the spectrum in the $\mathrm{DH}$ region (at $T_{\mathrm{H}}$ ). This fraction $p(\ll 1)$ is determined by the interaction, or the plasmon eigenmodes of the system (see Sec. IV). The majority of the spectrum should reflect the base temperature $T_{\text {base }}$ of the system. This leads to a nontrivial binary spectrum in the $\mathrm{IH}$ regions, as shown in the right inset. The focus of this study is the stability of this unusual mixture during transport.

This experimental scheme can be understood as a spatial analog of quantum quench. This analogy is allowed because the plasmon transport is unidirectional from the injection point through the channels to the ohmic contacts at the ends of the channels, as is the flow of time in quantum quench (see discussions in Sec. VB). The initial state prepared as 


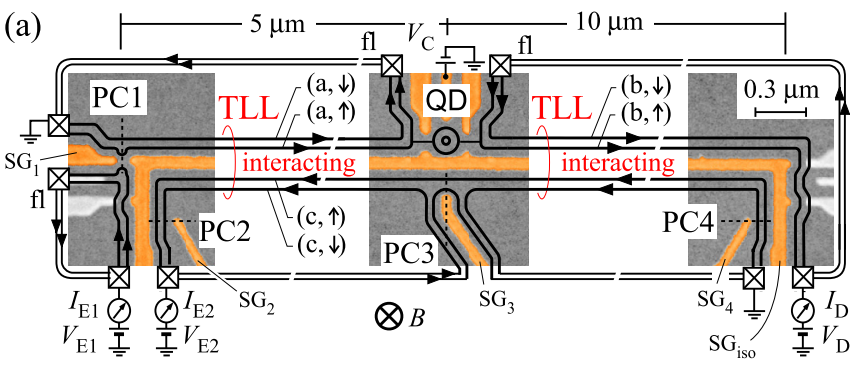

(b)
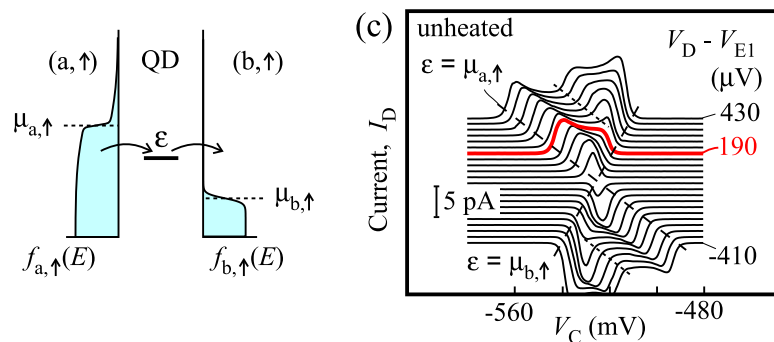

FIG. 2. (a) The measurement setup with three magnified scanning-electron micrographs with false color. The QD spectrometer and one of the heat injectors (PC1-PC4) are activated with the surface gates $\left(\mathrm{SG}_{\text {iso }}, \mathrm{SG}_{1-4}\right.$, etc.), while inactivated $\mathrm{PCs}$ are fully opened. The double lines indicate edge channels that form when PC1 and PC3 are activated, while only one of the PCs are activated in the actual measurements. The outer and inner channels serve spin-up and -down transport, respectively. The QD with a few electrons shows a charging energy of about $2 \mathrm{meV}$. Terminals labeled "fl" are floating ohmic contacts. (b) A schematic energy diagram of the transport through a QD level $\varepsilon$. Electronic spectra $f_{\mathrm{a}, \uparrow}(E)$ and $f_{\mathrm{b}, \uparrow}(E)$ can be determined from the current $I(\varepsilon)$. (c) Current spectra of the QD for various effective bias voltages $V_{\mathrm{D}}-V_{\mathrm{E} 1}$ taken with all PCs (including PC1) fully opened $\left(G_{i} / G_{\mathrm{q}}=2\right)$. Dashed lines indicate the energy alignment of the ground state with the chemical potential of the channels $\left(\varepsilon=\mu_{\mathrm{a} / \mathrm{b}, \uparrow}\right)$, while dotted lines indicate that of the excited states. Each trace is offset for clarity.

single electron excitations relaxes into plasmon excitations in the DH and IH regions. Such spatial evolution can be studied by using PC injectors at various distances from the QD spectrometer [20]. If the ergodicity is assumed, a many-body state may relax to a thermal-equilibrium state after traveling a long distance. However, "nonequilibrium steady state," which can be defined as a state that remains nonequilibrium even after traveling a sufficiently long (conceptually infinite) distance, may emerge in an integrable model. The comparison between the experiment in Sec. III and the simulation in Sec. IV implies that the binary spectrum is the signature of "nonequilibrium steady state" expected in the integrable model of TL liquid.

\section{EXPERIMENT}

\section{A. Sample and measurement technique}

The measurements were performed with an $\mathrm{AlGaAs} / \mathrm{GaAs}$ modulation-doped heterostructure in a perpendicular magnetic field of $5.9 \mathrm{~T}(v=2)$ at the lattice temperature of 20 $100 \mathrm{mK}$. The unprocessed 2DES has the electron density $2.9 \times$ $10^{11} \mathrm{~cm}^{-2}$ and the zero-field mobility $1.6 \times 10^{6} \mathrm{~cm}^{2} / \mathrm{Vs}$. The device shown in Fig. 2(a) involves split gates SG's to define the channels, PC charge injectors, and a QD spectrometer.
The quantized Hall resistance at $h / 2 e^{2}$ and the vanishing longitudinal resistance of the device ensure the formation of two chiral edge channels dominating the transport.

As shown in Fig. 2(a), three sections of edge channels $(\eta, \sigma)$, where $\eta$ specifies the region "a," "b," and "c," are defined by using the isolation gate $\mathrm{SG}_{\text {iso }}$ (width $w=0.1 \mu \mathrm{m}$ ) and a QD spectrometer inserted between the sections "a" and "b." Spin-full TL liquids labeled TLLs are formed in the interacting regions of $(\mathrm{a}, \sigma)$ and $(\mathrm{c}, \sigma)$ for the length of $5 \mu \mathrm{m}$, as well as $(\mathrm{b}, \sigma)$ and $(\mathrm{c}, \sigma)$ of $10 \mu \mathrm{m}$. We investigate how the spectra of $(\mathrm{a}, \uparrow)$ and $(\mathrm{b}, \uparrow)$ in the vicinity of the QD change when heat current is injected at different locations with respect to the spectrometer. This is done by selectively activating one of the four PCs; that is, injection from PC1 (PC2-PC4) implies that the spectrometer is placed in the $\mathrm{DH}(\mathrm{IH})$ region(s). The tunneling probability $D_{i, \sigma}$ of $i$ th $\mathrm{PC}$ for spin $\sigma$ can be estimated from its conductance $G_{i}=G_{\mathrm{q}}\left(D_{i, \uparrow}+D_{i, \downarrow}\right)$ with $G_{\mathrm{q}}=\frac{e^{2}}{h}$, where $G_{1}=I_{\mathrm{E} 1} / V_{\mathrm{E} 1}$ for $\mathrm{PC} 1$ and $G_{i}=I_{\mathrm{E} 2} / V_{\mathrm{E} 2}$ for PC $i$ $(i=2,3$, and 4$)$ are obtained from the current $I_{\mathrm{E} 1 / 2}$-voltage $V_{\mathrm{E} 1 / 2}$ characteristics.

Figure 2(c) shows current spectrum with varying effective QD bias $V_{\mathrm{D}}-V_{\mathrm{E} 1}$ between the drain and the emitter [See Fig. 2(a) for their locations], taken without heating (PC1 fully opened and PC2-PC4 fully closed). Current steps associated with the transport through the ground and excited levels are shown by dashed and dotted lines, respectively, indicating the level spacing of about $200 \mu \mathrm{eV}$. In the following experiments, we keep the effective bias voltage below $200 \mu \mathrm{V}$ to probe only the transport through the ground level $\varepsilon$ as shown in Fig. 2(b).

When $\varepsilon$ is swept with the gate voltage $V_{\mathrm{C}}$, the QD current measures the electronic spectrum $f_{\mathrm{a} / \mathrm{b}, \uparrow}(E)$ of $\operatorname{channel}(\mathrm{a} / \mathrm{b}, \uparrow)$ via the relation

$$
I(\varepsilon)=I_{0}\left\{f_{\mathrm{a}, \uparrow}(\varepsilon)\left[1-f_{\mathrm{b}, \uparrow}(\varepsilon)\right]-f_{\mathrm{b}, \uparrow}(\varepsilon)\left[1-f_{\mathrm{a}, \uparrow}(\varepsilon)\right]\right\},
$$

where $I_{0}$ is the saturated current on the step. For a large positive bias, the second term of Eq. (1) describing the negative component of the current can be neglected. Then, the current profiles around the onsets $\varepsilon=\mu_{\mathrm{a}, \uparrow}$ (the left edge of the peak at a positive bias) and $\mu_{\mathrm{b}, \uparrow}$ (the right edge) measure the spectra $f_{\mathrm{a}, \uparrow}(E)$ and $1-f_{\mathrm{b}, \uparrow}(E)$, respectively. Therefore, electronic spectra $f_{\mathrm{a}, \uparrow}(E)$ and $f_{\mathrm{b}, \uparrow}(E)$ can be evaluated from a single current trace $I_{\mathrm{D}}\left(V_{\mathrm{C}}\right)$. The unheated spectrum in Fig. 2(c) can be fitted well by using the Fermi distribution

$$
f_{\mathrm{F}}(E ; T)=\left[e^{(E-\mu) / k_{\mathrm{B}} T}+1\right]^{-1}
$$

with the thermal energy $k_{\mathrm{B}} T_{\mathrm{a} / \mathrm{b}, \uparrow}$ and chemical potential $\mu_{\mathrm{a} / \mathrm{b}, \uparrow}$. The sharp onsets on both sides of the peak indicate the thermal energy $k_{\mathrm{B}} T_{\text {base }}=9-10 \mu \mathrm{eV}$ at the base temperature $T_{\text {base }}$ in both channels. The QD spectroscopy relies on transport through a single level. Therefore we restrict ourselves at low-excitation conditions where populations to the excited states can be neglected, unless otherwise stated.

\section{B. Spectrum in the $\mathrm{DH}$ region}

First, we investigate the spectrum in the DH region using PC1 located $\sim 5 \mu \mathrm{m}$ upstream of the QD. Figure 3(a) schematically shows the corresponding channel layout. The DH region is formed in $(\mathrm{a}, \uparrow)$ and $(\mathrm{a}, \downarrow)$ by injecting heat through PC1 with an excitation voltage $V_{\mathrm{E} 1}=-100 \mu \mathrm{eV}$. Nonequilibrium 
(a)

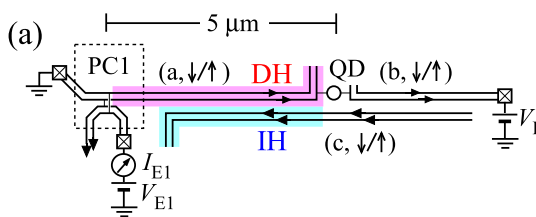

(b)
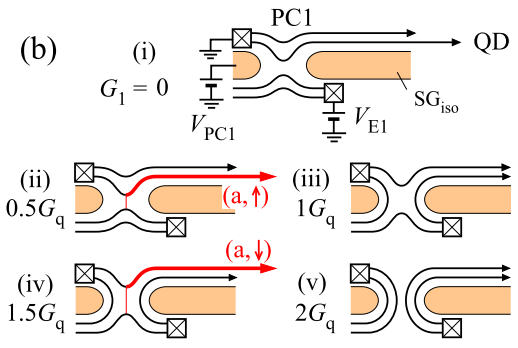
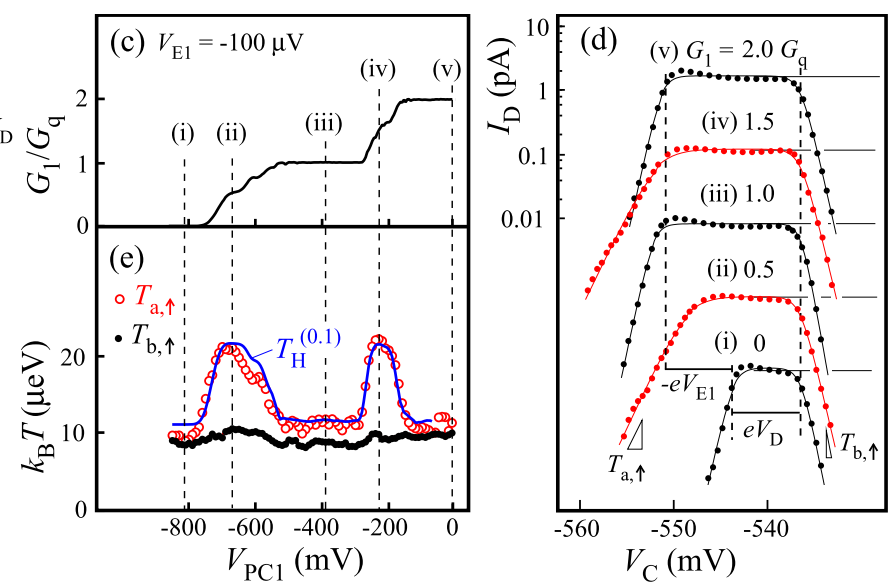

FIG. 3. (a) A schematic channel layout for measuring the spectrum in the DH region formed downstream of PC1 in (a, $\uparrow / \downarrow$ ). (b) Schematic channel geometries near PC1 for (i) $G_{1}=0$, (ii) $G_{1}=0.5 G_{\mathrm{q}}$, (iii) $G_{1}=G_{\mathrm{q}}$, (iv) $G_{1}=1.5 G_{\mathrm{q}}$, and (v) $G_{1}=2 G_{\mathrm{q}}$. Channels (a, $\uparrow$ ) and (a, $\downarrow$ ) can be heated in (ii) and (iv), respectively. (c) $V_{\mathrm{PC} 1}$ dependence of $G_{1} / G_{\mathrm{q}}$, measured by the current $I_{\mathrm{E} 1}$ at the excitation voltage $V_{\mathrm{E} 1}=-100 \mu \mathrm{V}$. (d) Current spectra with a flat-top region of the width associated with the effective bias. Measured current (circles) is fitted well with the Fermi distribution function (solid lines). The broadened edges measure the electron temperatures $T_{\mathrm{a} / \mathrm{b}, \uparrow}$ in the channels. The $I_{\mathrm{D}}$ and $V_{\mathrm{C}}$ scales apply to the trace $(\mathrm{v})$, while other traces are offset for clarity. (e) $k_{\mathrm{B}} T_{\mathrm{a} / \mathrm{b}, \uparrow}$ as a function of $V_{\mathrm{PC} 1} . T_{\mathrm{H}}^{(0.1)}$ is the expected temperature considering the coupling $p=0.1$. Small difference between $T_{\mathrm{a}, \uparrow}$ and $T_{\mathrm{b}, \uparrow}$ at unheated conditions (iii) and (v) might come from external noise in the ammeter for $I_{\mathrm{E} 1 \text {. }}$

charge can be injected selectively into (a, $\uparrow$ ) for the PC1 conductance $G_{1}$ in the range of $0<G_{1}<G_{\mathrm{q}}$ as shown in panel (ii) of Fig. 3(b), and (a, $\downarrow$ ) for $G_{\mathrm{q}}<G_{1}<2 G_{\mathrm{q}}$ in panel (iv). This was confirmed by measuring clear conductance steps in the split-gate voltage $V_{\mathrm{PC} 1}$ dependence of $G_{1}\left(=I_{\mathrm{E} 1} / V_{\mathrm{E} 1}\right)$ in Fig. 3(c).

Figure 3(d) summarizes the current spectra of the QD (filled circles) for various $G_{1}$ values, plotted as a function of $V_{\mathrm{C}}$. The QD spectrometer is operated at $V_{\mathrm{D}}=100 \mu \mathrm{eV}$. As the PC1 opening is increased from $G_{1} / G_{\mathrm{q}}=0$ to 2 , the width of the spectrum grows from $e V_{\mathrm{D}}=100 \mu \mathrm{eV}$ [trace (i)] to $e\left(V_{\mathrm{D}}-V_{\mathrm{E} 1}\right)=200 \mu \mathrm{eV}$ [traces (iii)-(v)], reflecting the channel (a, $\uparrow$ ) being switched from the grounded ohmic contact to the one biased at $V_{\mathrm{E} 1}$. Heating in (a, $\left.\uparrow\right)$ is manifested in the slope on the left edge of the spectra, which becomes distinctly gentle, indicating larger $T_{\mathrm{a}, \uparrow}$, in the tunneling regimes at $G_{1} / G_{\mathrm{q}}=0.5$ [trace (ii)] and 1.5 [trace (iv)]. Note that heating in $(\mathrm{a}, \uparrow)$ is resolved even when nonequilibrium charge is injected into (a, $\downarrow$ ). This ensures the coupling between the two channels. No heating is seen at quantized conductance $G_{1} / G_{\mathrm{q}}=1$ [trace (iii)] and 2 [trace (v)]. In contrast to the left edge of the spectra, the right edge, which probes (b, $\uparrow$ ), remains steep with $T_{\mathrm{b}, \uparrow} \simeq T_{\text {base }}$ for all $G_{1}$ values. This is reasonable, as no heating is expected for $(\mathrm{b}, \uparrow)$ in this channel layout [see Fig. 3(a)].

All the spectra in Fig. 3(d) can be fitted well with the Fermi distribution as shown by the solid lines. Figure 3(e) plots $k_{\mathrm{B}} T_{\mathrm{a}, \uparrow}$ (open circles) and $k_{\mathrm{B}} T_{\mathrm{b}, \uparrow}$ (solid circles) deduced from the fits, revealing significant heating in (a, $\uparrow$ ), for both injection into (a, $\uparrow)\left(G_{1} / G_{\mathrm{q}} \sim 0.5\right.$ at $\left.V_{\mathrm{PC} 1} \sim-660 \mathrm{mV}\right)$ and $(\mathrm{a}, \downarrow)\left(G_{1} / G_{\mathrm{q}} \sim 1.5\right.$ at $\left.V_{\mathrm{PC} 1} \sim-220 \mathrm{mV}\right)$. The fact that $T_{\mathrm{a}, \uparrow}$ reaches almost the same maximum values at $G_{1} / G_{\mathrm{q}} \sim 0.5$ and 1.5 indicates that the injected heat is equally partitioned between $(\mathrm{a}, \uparrow)$ and $(\mathrm{a}, \downarrow)$. As these data show, the coupling between hot and cold copropagating channels results in spectra that looks like the equilibrium Fermi distribution in both of the copropagating channels. Theory suggests that the actual distribution is different from the Fermi distribution even in the DH region [26,27]. However, the deviation is too small to be resolved experimentally, resulting in seemingly thermalized spectra indistinguishable from the equilibrium distribution. Consequently, the DH scheme is not suitable for studying nonequilibrium steady states.

\section{Spectrum in the $\mathrm{IH}$ region}

In contrast to the case of $\mathrm{DH}$ region, qualitatively different tunneling spectra emerge when the spectrometer is placed in the IH region. IH regions appear in $(\mathrm{a}, \sigma)$ and/or $(\mathrm{b}, \sigma)$ when one of PC2-PC4 is adjusted in the tunneling regime with the PC conductance $G_{i}=I_{\mathrm{E} 2} / V_{\mathrm{E} 2} \sim 0.5 G_{\mathrm{q}}$. Figure 4 summarizes the current spectra (solid circles) with $\mathrm{PC} 2$ in (a), PC3 in (b), and PC4 in (c). As the bias $V_{\mathrm{E} 2}$ of the PC is increased, an additional small but long tail develops, resulting in an anomalous spectrum that cannot be fitted with a single Fermi distribution function. The long tails appear on one or both sides of the peak depending on the location of the spectrometer with respect to the $\mathrm{PC}$.

For example, injection from $\mathrm{PC} 2$ induces an $\mathrm{IH}$ region in $(\mathrm{a}, \sigma)$ but not in $(\mathrm{b}, \sigma)$ [inset of Fig. 4(a)]. This is consistent with the observation that a tail appears only on the left side of the peak [anomalous excitation in $(\mathrm{a}, \uparrow)]$, by recalling that the current profiles on the left and right sides of the peak reflect $f_{\mathrm{a}, \uparrow}(E)$ and $1-f_{\mathrm{b}, \uparrow}(E)$, respectively.

Injection from $\mathrm{PC} 3$ should also induce an $\mathrm{IH}$ region only in $(\mathrm{a}, \sigma)$. This is consistent with the tail appearing on the left side [excitation in (a, $\uparrow)$ ] as seen in Fig. 4(b). The amplitude of the tail on the left side is comparable to that in Fig. 4(a). A small steplike profile on the right side is associated with the excited state of the QD, where electromagnetic and/or phonon-mediated energy transfer might be responsible only for this geometry with a short distance between PC3 and QD 




FIG. 4. Binary tunneling spectra in the IH regions with heat current injected from (a) PC2, (b) PC3, and (c) PC4. Measured current (circles) is fitted well with the binary spectrum (solid lines) with two temperatures, $T_{\mathrm{a}, \uparrow}^{(\mathrm{H})}$ and $T_{\mathrm{a}, \uparrow}^{(\mathrm{L})}$, coexisting in the channel. Each inset shows the schematic channel geometry with the location of IH regions of interest.

$(<0.3 \mu \mathrm{m})$ [38]. Therefore this steplike structure is disregarded in the following discussion.

Injection from PC4 induces $\mathrm{IH}$ regions in both $(\mathrm{a}, \sigma)$ and $(\mathrm{b}, \sigma)$, resulting in tails on both sides [excitation in (a, $\uparrow)$ and (b, $\uparrow)]$ in Fig. 4(c). In this case, PC4 excites left moving plasmons in modes [L, C/S], which drag nonequilibrium charges in $(b, \sigma)$ up to the right of the QD. This gives a tail on the right side of the peak. However, this measurement point is ill defined as it is located near the boundary between the $\mathrm{IH}$ region and the unheated upstream region of $(\mathrm{b}, \sigma)$. This could be the reason of the small amplitude of the right tail as compared to the tails in Figs. 4(a) and 4(b). Moreover, the left moving plasmons in the interacting channels $(\mathrm{b}, \sigma)$ and $(\mathrm{c}, \sigma)$ are interrupted by the QD and scatter into the other interacting region with channels $(\mathrm{a}, \sigma)$ and $(\mathrm{c}, \sigma)$. This plasmon scattering leads another $\mathrm{IH}$ region [denoted by $\mathrm{IH}^{\prime}$ in the inset of Fig. 4(c)] in $(\mathrm{a}, \sigma)$, which generates a tail on the left side of the peak. The small amplitude of the left tail reflects this plasmon scattering.

Apart from the quantitative differences, the unusual tails appear in all of the IH regions we investigated. As shown by thin solid lines in Fig. 4, all the spectra in the IH regions can be fitted well with the binary spectrum

$$
f_{\eta, \sigma}(E)=(1-p) f_{\mathrm{F}}\left(E ; T_{\eta, \sigma}^{(\mathrm{L})}\right)+p f_{\mathrm{F}}\left(E ; T_{\eta, \sigma}^{(\mathrm{H})}\right),
$$

which consists of majority carriers at lower temperature $T_{\eta, \sigma}^{(\mathrm{L})}$ and minority carriers of a fraction $p(\sim 0.1)$ at high temperature $T_{\eta, \sigma}^{(\mathrm{H})}$.

To further demonstrate the validity of Eq. (3), we examine the case of large excitation voltage, where the second term of Eq. (1) cannot be neglected. We show in Fig. 5 a spectrum excited using PC2 under a large excitation voltage of $V_{\mathrm{E} 2}=$ $2 \mathrm{meV}$ [panel (i)] and compare it with that without heating [panel (ii)], plotted in a linear scale. As already shown in Fig. 4(a), in this configuration heating occurs only in $(\mathrm{a}, \sigma)$ near the QD, which affects only the left side of the peak at low bias. In contrast, the spectrum in panel (i) of Fig. 5 shows a small negative tail (blue hatched region) on the right side, in addition to the tail on the left side (red hatched region). This signifies the presence of hot holes as well as hot electrons in (a, $\uparrow)$. The whole spectrum including the negative tail is reproduced well with Eq. (3) as shown by the solid line. The distribution functions $f_{\mathrm{a} \uparrow}(E)$ and $1-f_{\mathrm{b}, \uparrow}(E)$ deduced from the fit are shown by solid lines in the inset, highlighting the anomalous binary spectrum $f_{\mathrm{a}, \uparrow}(E)$ compared to the Fermi distribution function $f_{\mathrm{F}}(E)$ shown by a dashed line.

Binary spectrum can be seen in a wide range of gate voltage, $V_{\mathrm{PC} i}$, for $i$ th $\mathrm{PC}$ with $i=2,3$, and 4 . Figure 6 summarizes the $V_{\mathrm{PC} 2}$ dependence of the PC2 conductance in (a) and the fitting parameters, the fraction $p$ in (b), and two distinct temperatures $T_{\mathrm{a}, \uparrow}^{(\mathrm{H})}$ (circles) and $T_{\mathrm{a}, \uparrow}^{(\mathrm{L})}$ (black solid line) in (c), for the binary spectrum. At this large bias voltage $V_{\mathrm{E} 2}=1 \mathrm{mV}, G_{2}$ in Fig. 6(a) does not show quantized conductance at $G_{\mathrm{q}}$, which gives ambiguity in the estimate of $D_{2, \sigma}$. The binary spectrum with high $T_{\mathrm{a}, \uparrow}^{(\mathrm{H})}$ is seen in the entire tunneling regime, while $T_{\mathrm{a}, \uparrow}^{(\mathrm{L})}$ as well as $T_{\mathrm{b}, \uparrow}$ in the unheated region remain low comparable to the base temperature.

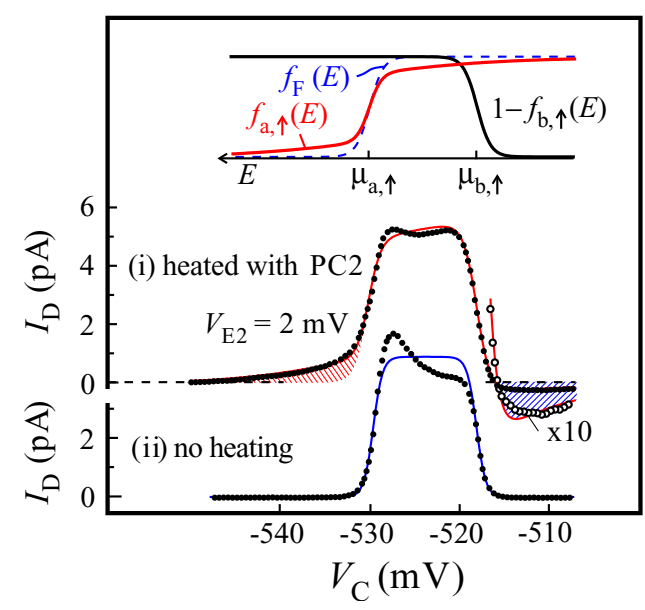

FIG. 5. Current spectrum in a linear scale (i) with heat injection from PC2 and (ii) without heat injection. The positive and negative current tails in the red and blue hatched regions in (i) represent the excitation of hot electrons and hot holes, respectively, consistent with the binary spectrum (the solid lines). The deduced distribution functions $f_{\mathrm{a}, \uparrow}(E)$ and $1-f_{\mathrm{b}, \uparrow}(E)$ are shown in the inset. The binary spectrum in $f_{\mathrm{a}, \uparrow}(E)$ contrasts with a single Fermi distribution function $f_{\mathrm{F}}(E)$ (dashed line). 


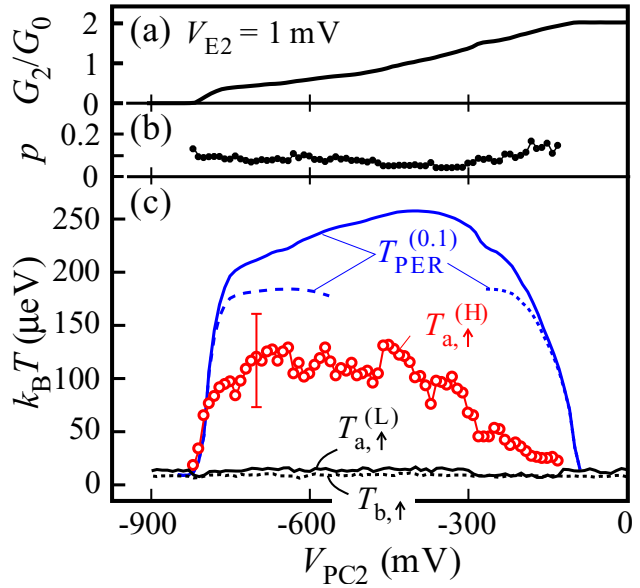

FIG. 6. (a) Conductance of $\mathrm{PC} 2, G_{2}=I_{\mathrm{E} 2} / V_{\mathrm{E} 2}$, as a function of the gate voltage, $V_{\mathrm{PC} 2}$. (b and c) $V_{\mathrm{PC} 2}$ dependence of the fraction $p$ of hot minority carriers in (b) and thermal energies for $T_{\mathrm{a}, \uparrow}^{(\mathrm{H})}$ (circles), $T_{\mathrm{a}, \uparrow}^{(\mathrm{L})}$ (black solid line), and $T_{\mathrm{b}, \uparrow}$ (black dashed line) in (c) deduced from the fitting. The parameter $T_{\mathrm{a}, \uparrow}^{(\mathrm{H})}$ is smaller than but comparable to $T_{\mathrm{PER}}^{(0.1)}$, which is expected in the assumption of reaching an identical temperature. The blue solid, dashed, and dotted lines are $T_{\mathrm{PER}}^{(0.1)}$ by assuming $D_{2, \uparrow}=D_{2, \downarrow}, D_{2, \downarrow}=0$, and $D_{2, \uparrow}=0$, respectively.

Strikingly, the observed binary spectrum did not decay into a single Fermi function for any geometries we examined. The factor $p(\sim 0.1)$ is found to be comparable for various distances from the $\mathrm{PC}$ to the $\mathrm{QD}$, including the shortest case with PC3 [Fig. 4(b)], $5 \mu \mathrm{m}$ with PC2 [Fig. 4(a)], and $10 \mu \mathrm{m}$ with PC4 in the reversed magnetic field (see Appendix B and Fig. 10). These distances are much longer than $l_{\mathrm{el}}\left(\sim 0.1 \mu \mathrm{m}\right.$ at $\left.V_{\mathrm{E}}=1 \mathrm{meV}\right)$ for electronic relaxation to plasmons. Therefore, the observed binary spectrum supports the picture of noninteracting plasmon excitations and the nonthermalizing character of the $1 \mathrm{D}$ edge channels.

\section{Comparison of the spectra}

The spectra in the $\mathrm{DH}$ and $\mathrm{IH}$ regions are compared in the same range of excitation voltages in Fig. 7, where the horizontal axis is taken as the dot energy $\varepsilon$ relative to $\mu_{\mathrm{a}, \uparrow}$. The spectrum for the DH region at $G_{1}=1.5 G_{\mathrm{q}}$ in Fig. 7 (a) is measured at various $V_{\mathrm{E} 1}$ by keeping the effective bias constant $\left(V_{\mathrm{E} 1}-V_{\mathrm{D}}=200 \mu \mathrm{V}\right)$. Heating in $(\mathrm{a}, \uparrow)$ is seen as broadening on the left edge of the peak. The linear slope over a few orders of magnitude in the logarithmic scale of $I_{D}$ suggests that all carriers in the $\mathrm{DH}$ region can be characterized by a single temperature $T^{(\mathrm{DH})}$. In contrast, the unusual tail for the IH region, which can be recognized from $V_{\mathrm{E} 2} \sim 200 \mu \mathrm{V}$ and becomes prominent at $V_{\mathrm{E} 2} \geqslant 500 \mu \mathrm{V}$ in Fig. 7(b), can be characterized by the binary spectrum with parameters $T^{(\mathrm{H})}$ (gentle slope), $T^{(\mathrm{L})}$ (steep slope), and $p$.

One can see that $T^{(\mathrm{DH})}$ in the $\mathrm{DH}$ region [Fig. 7(a)] is somewhat higher but comparable to $T^{(\mathrm{H})}$ in the $\mathrm{IH}$ region [Fig. 7(b)], by comparing the spectra at the same excitation voltages $\left(V_{\mathrm{E} 1}=V_{\mathrm{E} 2} \sim 600 \mu \mathrm{V}\right)$. This implies the strong relation between the two spectra. Based on the argument in Sec. II B, the different spectra in the $\mathrm{DH}$ and $\mathrm{IH}$ regions can be understood by considering the amplitudes of the plasmon

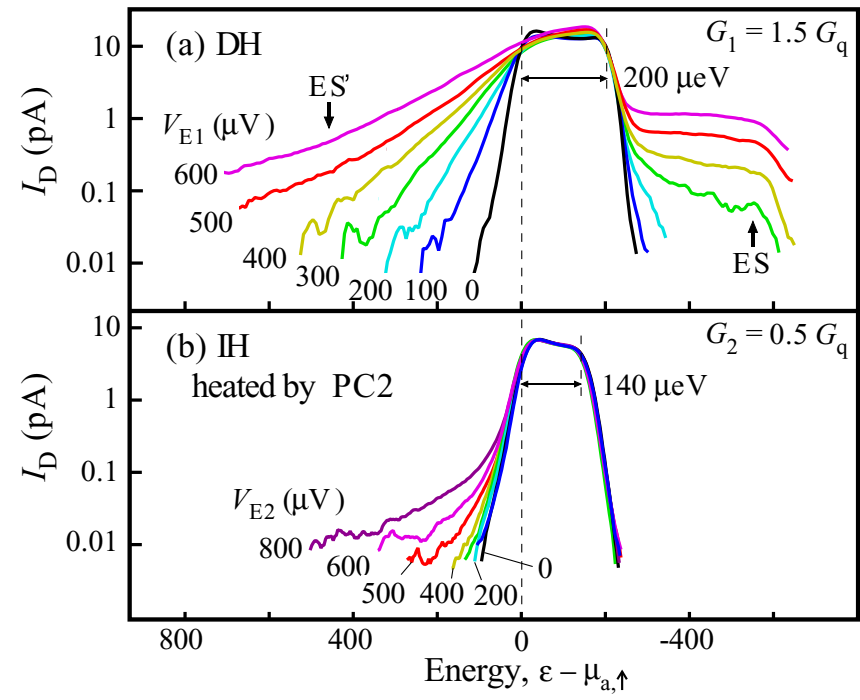

FIG. 7. (a) Tunneling spectra measured in the $\mathrm{DH}$ region induced by $\mathrm{PC} 1$ at $G_{1}=1.5 G_{\mathrm{q}}$. Its $V_{\mathrm{E} 1}$ dependence is taken by changing both $V_{\mathrm{E} 1}$ and $V_{\mathrm{D}}$ to keep $V_{\mathrm{E} 1}-V_{\mathrm{D}}=200 \mu \mathrm{V}$. Excited-state features are indicated by arrows (ES and $\left.\mathrm{ES}^{\prime}\right)$. (b) Tunneling spectra measured in the $\mathrm{IH}$ region induced by $\mathrm{PC} 2$ at $G_{2}=0.5 G_{\mathrm{q}}$. The binary spectrum develops with increasing $V_{\mathrm{E} 2}$.

excitations. The channels in the DH region are full of hot plasmons with large amplitudes as shown in Fig. 1(b), which seemingly looks like a thermalized state when the system is measured with a QD spectrometer. In contrast, the channels in the IH region involve small amplitudes of hot plasmons in the background of cold plasmons, which could lead to the anomalous binary spectrum.

We should add some comments on the effect of excited states in the dot. In Fig. 7(a), a faint kink marked by ES' and a steplike structure marked by ES appear when the excitation voltage $V_{\mathrm{E} 1}$ exceeds the typical level spacing (about $200 \mu \mathrm{V}$ ) of the QD. The former $\left(\mathrm{ES}^{\prime}\right)$ can be understood as hot electrons passing through an excited state, and the latter (ES) can also be attributed to a complex excitation process to an excited state. Both of these additional features start to appear at $V_{\mathrm{E} 1}$ values much higher than that at which the heating in the channel starts to be visible. In contrast, no features associated with the excited states appear for the IH region, unless $V_{\mathrm{E} 2}$ exceeds $2 \mathrm{mV}$. This is presumably due to the weak coupling $(p \sim 0.1)$ between the $\mathrm{DH}$ and $\mathrm{IH}$ regions.

\section{E. Heat flow}

Provided that the binary distribution of Eq. (3) holds well, the temperatures in $\mathrm{DH}$ and $\mathrm{IH}$ regions can be related to each other by considering the heat flow in the system. The heat current injected from the $i$ th $\mathrm{PC}$ is given by $W_{i}=\frac{1}{2} G_{\mathrm{q}} \sum_{\sigma} D_{i, \sigma}\left(1-D_{i, \sigma}\right) V_{\mathrm{E}}^{2}$ with the partitioning factor $D(1-D)[16,20]$. This heat should be redistributed over the four channels. As discussed in Sec II B, excitations in $\mathrm{DH}$ and $\mathrm{IH}$ regions are expected to be correlated. For simplicity, we assume that all carriers in the $\mathrm{DH}$ region and the fraction $p$ of carriers in the IH region share an identical temperature $T_{\mathrm{H}}^{(p)}$ while the cold majority $(1-p)$ in the $\mathrm{IH}$ region remains 

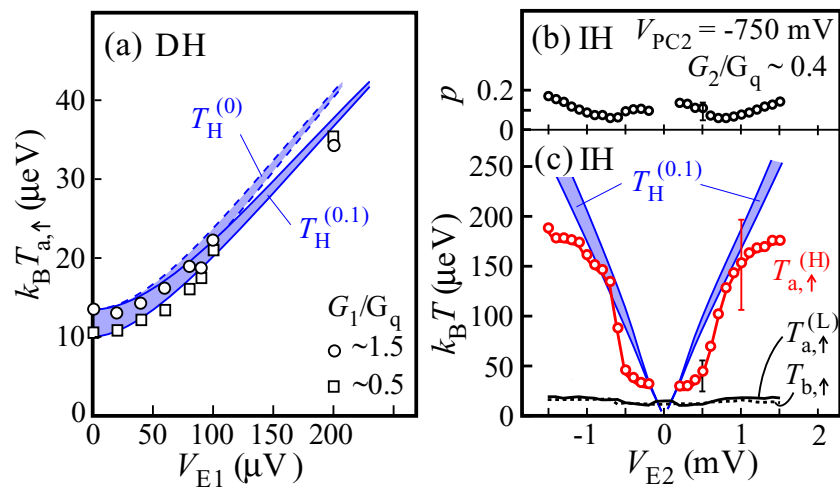

FIG. 8. (a) The bias voltage $V_{\mathrm{E} 1}$ dependence of $k_{\mathrm{B}} T_{\mathrm{a}, \uparrow}$ obtained at $G_{2} / G_{\mathrm{q}} \sim 1.5$ (circles) and $\sim 0.5$ (squares). Solid and dashed lines show $T_{\mathrm{H}}^{(0.1)}$ and $T_{\mathrm{H}}^{(0)}$, respectively, considering the variation of $k_{\mathrm{B}} T_{\text {base }}=10$ (lower bounds) and $13 \mu \mathrm{eV}$ (upper bounds). (b and c) The bias voltage $V_{\mathrm{E} 2}$ dependence of $p$ in (b) and $k_{\mathrm{B}} T_{\mathrm{a}, \uparrow}^{(\mathrm{H})}$, $k_{\mathrm{B}} T_{\mathrm{a}, \uparrow}^{(\mathrm{L})}$, and $k_{\mathrm{B}} T_{\mathrm{b}, \uparrow}$ in (c). The error bars show typical ambiguity in fitting the binary spectrum. Blue solid lines show $T_{\mathrm{H}}^{(0.1)}$, considering the ambiguity of tunneling probabilities between $D_{2, \uparrow}=D_{2, \downarrow}=$ $G_{2} / 2 G_{\mathrm{q}}$ (upper bound) and $D_{2, \uparrow}=G_{2} / G_{\mathrm{q}}$ and $D_{2, \downarrow}=0$ (lower bound).

at $T_{\text {base }}$. This assumption can be justified for the high-energy spectrum $\left(\left|\varepsilon-\mu_{\mathrm{a}, \uparrow}\right| \gg k_{\mathrm{B}} T_{\mathrm{H}}^{(p)}\right)$ in the weak coupling limit $(p \ll 1)$ of the quantum quench problem shown in Sec. IV B. This crude approximation allows us to compare the different experiments for the $\mathrm{DH}$ and $\mathrm{IH}$ regions. Then, the energy conservation law suggests that the spectra are expected to share the same temperature given by $T_{\mathrm{H}}^{(p)}=\sqrt{T_{\text {base }}^{2}+\frac{3 h}{(1+p) \pi^{2} k_{\mathrm{B}}^{2}} W_{i}}$.

Figure 8(a) shows the bias-voltage $V_{\mathrm{E} 1}$ dependence of the deduced thermal energy $k_{\mathrm{B}} T_{\mathrm{a}, \uparrow}$ in the $\mathrm{DH}$ region taken at $G_{2} / G_{\mathrm{q}} \sim 1.5$ (circles) and $\sim 0.5$ (squares). Figures 8 (b) and 8(c) summarize $V_{\mathrm{E} 2}$ dependence of the fitting parameters [ $p$ in (b) and $k_{\mathrm{B}} T_{\mathrm{a}, \uparrow}^{(\mathrm{H})}$ (open circles), $k_{\mathrm{B}} T_{\mathrm{a}, \uparrow}^{(\mathrm{L})}$ (solid line) and $k_{\mathrm{B}} T_{\mathrm{b}, \uparrow}$ (dotted line) in (c)] for the IH region induced by $\mathrm{PC} 2$. Here we have restricted the ranges $V_{\mathrm{E} 1} \leqslant 0.2 \mathrm{mV}$ and $V_{\mathrm{E} 2} \leqslant 1.5 \mathrm{meV}$, where excited states in the QD play no visible effects in the spectrum. By choosing a typical value of $p=0.1$ from Fig. 8(b), one can see that $k_{\mathrm{B}} T_{\mathrm{a}, \uparrow}$ in Fig. 8(a) as well as $k_{\mathrm{B}} T_{\mathrm{a}, \uparrow}^{(\mathrm{H})}$ in Fig. 8(c) follow reasonably well the same form of $T_{\mathrm{H}}^{(0.1)}$. This suggests that the carriers in the DH region and the minority carriers in the IH region indicate a similar spectrum. Quantitative disagreement, such as the deviation of $k_{\mathrm{B}} T_{\mathrm{a}, \uparrow}^{(\mathrm{H})}$ from a linear dependence in Fig. 8(c), might come from this crude approximation and possible energy-dependent coupling between the channels [25]. Note that the temperature $T_{\mathrm{a}, \uparrow}^{(\mathrm{L})}$ [solid line in Fig. 8(c)] of the majority carriers always remains cold. Therefore, the total energy is well conserved in the four channels with negligible heat leakage to other degrees of freedom, which ensures that the channels are effectively isolated from the environment. The same temperature $T_{\mathrm{H}}^{(0.1)}$ is also plotted in Fig. 6(c), where one can see that the observed $T_{\mathrm{a}, \uparrow}^{(\mathrm{H})}$ is smaller but comparable to $T_{\mathrm{H}}^{(0.1)}$. These evidences support that the excitations in DH and IH regions are strongly correlated.
Note that the above analysis is based on the assumption where the energy distribution can be approximated by Fermi distribution in the DH region and the binary distribution in the IH region. Rigorous analysis should be made with the TL model, which is beyond the scope of this paper. However, simulations of quantum quench, a time-dependent problem for initial states prepared at different temperatures, strongly support this approximation, as shown in the next section.

\section{SIMULATIONS WITH THE TL MODEL}

\section{A. Plasmon eigenmodes}

Plasmon modes in the edge channels can be conveniently described by a capacitance model [24,31]. Interaction inside or between quantum Hall edge channels can be parametrized by capacitances per unit length, $C_{\mathrm{ch}}$ for intrachannel coupling and $C_{i j}$ for interchannel coupling between $i$ th and $j$ th channel $[i, j \in\{(\mathrm{r}, \downarrow),(\mathrm{r}, \uparrow),(\ell, \uparrow),(\ell, \downarrow)\}]$. For simplicity, we only consider nearest neighbor couplings with $C_{\mathrm{X}}$ between copropagating channels and $C_{\mathrm{Z}}$ between counterpropagating channels, as shown in Fig. 1(a). We assumed that $C_{\mathrm{ch}}$ is identical for all channels. Excess charge density $\rho_{i}$ and potential $V_{i}$ of $i$ th channel are related by the coupling capacitances; $\rho_{i}=C_{i j} V_{j}$ with the capacitance matrix of the form

$$
\mathbf{C}=C_{\mathrm{ch}}\left(\begin{array}{cccc}
1+c_{\mathrm{X}} & -c_{\mathrm{X}} & 0 & 0 \\
-c_{\mathrm{X}} & 1+c_{\mathrm{X}}+c_{\mathrm{Z}} & -c_{\mathrm{Z}} & 0 \\
0 & -c_{\mathrm{Z}} & 1+c_{\mathrm{X}}+c_{\mathrm{Z}} & -c_{\mathrm{X}} \\
0 & 0 & -c_{\mathrm{X}} & 1+c_{\mathrm{X}}
\end{array}\right)
$$

in the order of the channel index $i=(\mathrm{r}, \downarrow),(\mathrm{r}, \uparrow),(\ell, \uparrow)$, and $(\ell, \downarrow)$. Here, $c_{\mathrm{X}}=C_{\mathrm{X}} / C_{\mathrm{ch}}$ and $c_{\mathrm{Z}}=C_{\mathrm{Z}} / C_{\mathrm{ch}}$ denote the normalized coupling strengths. Charge conservation in each channel can be represented by $\partial \rho_{i} / \partial t=-S_{i} G_{\mathrm{q}} \partial V_{i} / \partial x$ with the chirality $S_{(\mathrm{r}, \sigma)}=1$ for right-moving channels and $S_{(\ell, \sigma)}=-1$ for left-moving channels, and $G_{\mathrm{q}}=\frac{e^{2}}{h}$. Coupled wave equation of the plasmons can be written in the form

$$
\partial \rho_{i} / \partial t=-M_{i j} \partial \rho_{j} / \partial x
$$

with $M_{i j}=S_{i} G_{\mathrm{q}}\left\{\mathbf{C}^{-1}\right\}_{i j}$.

The plasmon eigenmodes can be obtained by diagonalizing the matrix M. The transformation matrix $\mathbf{T}$ that satisfies $\mathbf{T}^{\dagger} \mathbf{M T}=\operatorname{diag}\left(v_{m}\right)$ describes the plasmon eigenmode $\tilde{\rho}_{m}$ and the velocity $v_{m}$ for $m=[\mathrm{R}, \mathrm{C}],[\mathrm{R}, \mathrm{S}],[\mathrm{L}, \mathrm{S}]$, and [L,C]. For a weak coupling limit $c_{\mathrm{Z}} \ll c_{\mathrm{X}} \ll 1$, eigenmodes can be approximately written as

$$
\begin{gathered}
\tilde{\rho}_{\mathrm{R}, \mathrm{C}} \simeq\left(1,1-c_{\mathrm{Z}} / 2 c_{\mathrm{X}},-c_{\mathrm{Z}} / 2,0\right) / \sqrt{2} \\
\tilde{\rho}_{\mathrm{R}, \mathrm{S}} \simeq\left(-1,1+c_{\mathrm{Z}} / 2 c_{\mathrm{X}},-c_{\mathrm{Z}} / 2,0\right) / \sqrt{2} \\
\tilde{\rho}_{\mathrm{L}, \mathrm{S}} \simeq\left(0,-c_{\mathrm{Z}} / 2,1+c_{\mathrm{Z}} / 2 c_{\mathrm{X}},-1\right) / \sqrt{2} \\
\rho_{\mathrm{L}, \mathrm{C}} \simeq\left(0,-c_{\mathrm{Z}} / 2,1-c_{\mathrm{Z}} / 2 c_{\mathrm{X}}, 1\right) / \sqrt{2}
\end{gathered}
$$

with the velocity $v_{\mathrm{R} / \mathrm{L}, \mathrm{C}} \simeq \pm v_{0}\left(1-c_{\mathrm{Z}} / 2\right)$ for charge modes and $v_{\mathrm{R} / \mathrm{L}, \mathrm{S}} \simeq \pm v_{0}\left(1-2 c_{\mathrm{X}}-c_{\mathrm{Z}} / 2\right)$ for spin modes, where $v_{0}=G_{\mathrm{q}} / C_{\mathrm{ch}}$ is the uncoupled plasmon velocity in a single channel. These plasmon eigenmodes are schematically shown 
in Fig. 1(a). Note that these plasmon eigenmodes are fully consistent with the TL model, as shown in Sec. IV B.

When a unit charge is injected into $(\ell, \uparrow)$, it can be expressed as

$$
(0,0,1,0) \simeq \sqrt{2} s\left(\tilde{\rho}_{\mathrm{R}, \mathrm{C}}+\tilde{\rho}_{\mathrm{R}, \mathrm{S}}\right)+\left(\tilde{\rho}_{\mathrm{L}, \mathrm{C}}+\tilde{\rho}_{\mathrm{L}, \mathrm{S}}\right) / \sqrt{2}
$$

with a small fractionalization ratio $s=c_{\mathrm{Z}} / 4=C_{\mathrm{Z}} / 4 C_{\mathrm{ch}}$. This explains large and small plasmon amplitudes in the $\mathrm{DH}$ and $\mathrm{IH}$ regions, respectively, as schematically illustrated in Fig. 1(b).

The above capacitance model is convenient for describing different coupling parameters between the channels. If required, the capacitances can be obtained by solving electrostatic potential around the edge channels [24]. Actually, the model has been applied to reproduce plasmon transport in edge channels including plasmon interferrometers [31]. In this paper, we use the matrix $\mathbf{T}$ and parameters $c_{\mathrm{X}}$ and $c_{\mathrm{Z}}$ to solve the quench problem shown in the next subsection.

\section{B. Quantum quench}

We have experimentally studied how the energy spectrum changes along the interacting edge channels under continuously injecting nonequilibrium electrons from a PC (spatial analog of quantum quench). This can be compared with the standard quantum quench problem asking how the momentum spectrum changes with time in the interacting channels after preparing a nonequilibrium state. Here we apply the quench problem to the counterpropagating channels to see the emergence of a binary spectrum.

We followed the approach taken by Kovrizhin and Chalker to study the quench problem for two copropagating channels (see Refs. [14,36] and references therein). Here, four infinitely long channels with channel index $j(=1-4)$, i.e., two right movers $(j=1$ and 2$)$ and two left movers $(j=3$ and 4$)$, are initially prepared in independent thermal equilibrium at different temperatures $T_{j}\left(T_{1}=T_{3}=T_{4}=T_{\text {base }}\right.$ and $T_{2}=T_{\mathrm{H}}>$ $\left.T_{\text {base }}\right)$. The initial state of each channel at $t=0$ is described by the correlation function $\mathcal{G}_{j}(x ; t=0)=\mathcal{G}_{T_{j}}^{(\mathrm{eq})}(x)$, where $x$ is distance and $\mathcal{G}_{T}^{(\mathrm{eq})}(x)=\frac{i}{2 \hbar}\left(\pi k_{\mathrm{B}} T / \hbar v_{0}\right) / \sinh \left[\left(\pi k_{\mathrm{B}} T / \hbar v_{0}\right) x\right]$ is the correlation function in thermal equilibrium at temperature $T$. Corresponding momentum distribution function $f_{j}(k, t=0)$ is the Fermi distribution function $f_{T}^{(\mathrm{eq})}(k)=$ $1 /\left[\exp \left(\hbar v_{0} k / k_{\mathrm{B}} T\right)+1\right]$ at $T=T_{j}$.

Time evolution of the system is calculated based on the standard bosonization technique for the TL model [39]. The interaction parameters often described by $g$ 's are equivalent to those in the capacitance model in Sec. IV A; i.e., the socalled $g_{4}$ and $g_{2}$ parameters represent the diagonal and offdiagonal elements, respectively, of $\mathbf{C}^{-1}$. It should be noted that backscattering with the so-called $g_{1}$ parameter, which diverts the system away from the TL model, is well suppressed in the edge channels. The Bogoliubov transformation can also be performed with the same matrix $\mathbf{T}$. Then, the dynamics is simply described by noninteracting plasmon modes with constant velocities. For the independent initial states at $T_{j}$, the equal-time correlation function $\mathcal{G}_{j}(x ; t)$ at time $t$ can be described by a product of initial correlation functions $\mathcal{G}_{T_{j}}^{(\mathrm{eq})}(x)$ of all channels as

$$
\begin{aligned}
\mathcal{G}_{j}(x ; t)= & \prod_{j^{\prime}}\left[\mathcal{G}_{T_{j^{\prime}}}^{(\mathrm{eq})}(x)\right]^{p_{j j^{\prime}}} \\
& \times \prod_{m, m^{\prime}}\left[\frac{\mathcal{G}_{T_{j^{\prime}}}^{(\mathrm{eq})}\left(x+\Delta v_{m, m^{\prime}} t\right)}{\mathcal{G}_{T_{j^{\prime}}}^{(\mathrm{eq})}\left(\Delta v_{m, m^{\prime}} t\right)}\right]^{c_{j j^{\prime} m} c_{j j^{\prime} m^{\prime}}},
\end{aligned}
$$

with the power $p_{j j^{\prime}}=\sum_{m}\left|T_{m j} T_{m j^{\prime}}\right|^{2}$ that satisfies $\sum_{j^{\prime}} p_{j j^{\prime}}=$ 1. $c_{j j^{\prime} m}$ represents the coupling between the channels $j$ and $j^{\prime}$ through the plasmon mode $m$, and $\Delta v_{m, m^{\prime}}=v_{m^{\prime}}-v_{m^{\prime}}$ is the velocity difference between the modes $m$ and $m^{\prime}$. For the steady state, $\mathcal{G}_{j}(x ; \infty)$ at $t \rightarrow \infty$ is given by a power mean of initial correlation functions as

$$
\mathcal{G}_{j}(x ; \infty)=\prod_{j^{\prime}}\left[\mathcal{G}_{T_{j^{\prime}}}^{(\mathrm{eq})}(x)\right]^{p_{j j^{\prime}}} .
$$

For the weak coupling limit $\left(c_{\mathrm{Z}} \ll c_{\mathrm{X}} \ll 1\right)$, we have $p_{j j^{\prime}} \sim$ 0.5 between copropagating channels $\left(S_{j} S_{j^{\prime}}=1\right)$ while $p_{j j^{\prime}}$ $\left(\sim s^{2}\right) \ll 1$ between counterpropagating channels $\left(S_{j} S_{j^{\prime}}=\right.$ $-1)$. Finally, the momentum distribution function $f_{j}^{(\mathrm{st})}(k)$ is obtained from the Fourier transform of $\mathcal{G}_{j}(x, \infty)$.

When only one channel $(j=2)$ is heated to $T_{\mathrm{H}}$ with others kept at $T_{\text {base }}$ in the initial condition, Eq. (12) can be simplified as

$$
\mathcal{G}_{j}(x ; \infty)=\mathcal{G}_{T_{\text {base }}}^{(\mathrm{eq})}\left[\mathcal{G}_{T_{\mathrm{H}}}^{(\mathrm{eq})} / \mathcal{G}_{T_{\text {base }}}^{(\mathrm{eq})}\right]^{p_{j 2}} .
$$

Focusing on the correlation function at shorter distance $\left(|x| \lesssim \hbar v_{0} / k_{\mathrm{B}} T_{\mathrm{H}}\right)$ or the spectrum at larger momentum $(|k| \gtrsim$ $\left.k_{\mathrm{B}} T_{\mathrm{H}} / \hbar v_{0}\right)$, the ratio $X \equiv \mathcal{G}_{T_{\mathrm{H}}}^{(\mathrm{eq})} / \mathcal{G}_{T_{\text {base }}}^{(\mathrm{eq})} \sim 1$ to the power of $p \equiv p_{j 2} \ll 1$ for $j=3$ or 4 can be approximated to $X^{p} \simeq 1+$ $p(X-1)+O\left((X-1)^{2}\right)$. This yields $\mathcal{G}_{j} \simeq(1-p) \mathcal{G}_{T_{\text {base }}}^{\text {(eq) }}+$ $p \mathcal{G}_{T_{\mathrm{H}}}^{(\mathrm{eq})}$. Its Fourier spectrum exhibits a binary spectrum of the form

$$
f_{j=3,4}^{(\mathrm{st})}(k) \simeq(1-p) f_{T_{\text {base }}}^{(\mathrm{eq})}(k)+p f_{T_{\mathrm{H}}}^{(\mathrm{eq})}(k)
$$

in the counterpropagating channels ( $j=3$ or 4$)$. In this way, the appearance of an approximate binary spectrum is suggested in the quantum quench problem. In contrast, electronic spectrum in the copropagating channels $(j=1$ and 2) can be approximated to $f_{j=1,2}^{(\mathrm{st})}(k) \simeq f_{T_{\mathrm{H}}}^{(\mathrm{eq})}(k)$ by neglecting an anomaly around $k=0$. Therefore, the two spectra in counterand copropagating channels share an identical spectrum. This characteristics is used in the analysis of heat flow in Sec. III E.

The exact solution of the spectra are neither the binary spectrum nor the Fermi distribution function. The accurate spectrum can be obtained by performing a Fourier transform of Eq. (13). The solid lines in Figs. 9(a) and 9(b) show the initial momentum distribution functions $f_{j}^{(\text {ini) }}(k)$ and the steady-state distribution function $f_{j}^{(\mathrm{st})}$, respectively, plotted as a function of the normalized momentum $\hbar v_{0} k / k_{\mathrm{B}} T_{\text {base }}$. Here, we assumed $T_{\mathrm{H}}=10 T_{\text {base }}$ and coupling parameters $c_{\mathrm{X}}=0.8$ and $c_{\mathrm{Z}}=0.6$. The parameters, which are realistic in the capacitance model in Sec. IV A, were chosen to imitate the binary spectra in Fig. 4. The copropagating channels 1 and 2 resulted in similar spectra $\left(f_{1} \sim f_{2}\right)$, which resemble a single Fermi distribution function shown by the dashed line. In contrast, anomalous spectra $\left(f_{3}\right.$ and $\left.f_{4}\right)$ emerge in the counterpropagating channels 


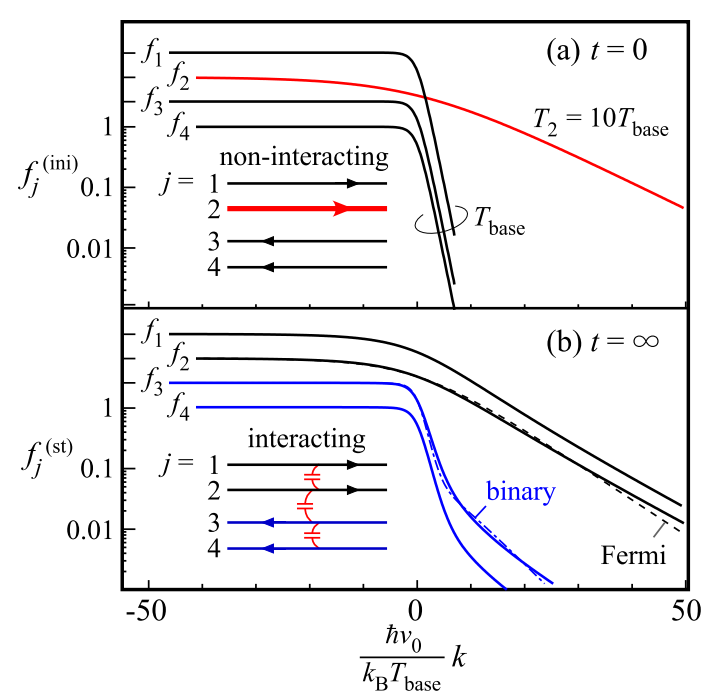

FIG. 9. Momentum distribution functions for (a) initial states in noninteracting channels and (b) steady state at $t=\infty$ after the interaction is turned on at $t=0$. Each trace is offset for clarity. Initially, channels are in thermal equilibrium at $T_{1}=T_{3}=T_{4}=T_{\text {base }}$ and $T_{2}=10 T_{\text {base }}$ in (a). Nonequilibrium steady states are reached at $t=\infty$ in (b), where quasibinary spectrum emerges in counterpropagating channels 3 and 4 . The dashed and dash-dotted lines show single and binary Fermi distribution functions, respectively, adjusted to the spectra based on the TL theory (solid lines). The schematic channel geometries without and with interaction are shown in the inset of (a) and (b), respectively.

3 and 4 . The spectrum can be approximated by a binary spectrum composed of hot and cold carriers, as shown by the dash-dotted line. In this range of plots in Fig. 9(b), the temperature (the inverse of the slope) of hot minority carriers in the counterpropagating channels is somewhat lower than that in copropagating channels.

In this way, the unusual binary spectrum can emerge in quantum-quench simulation. The simulation can be compared to the experiment by regarding the heated channel $(j=2)$ as $(\ell, \uparrow)$ in Fig. 1(b), spectra $f_{1}$ and $f_{2}$ as those in the DH region, and spectra $f_{3}$ and $f_{4}$ as those in the IH region.

\section{DISCUSSION}

\section{A. Coupling strength}

The factor $p$ deduced from the fits, which ranges from 0.03 to 0.2 in our PCs, measures the coupling between the counterpropagating channels across the isolation gate $\left(G_{\text {iso }}\right)$ of the width $w \sim 0.1 \mu \mathrm{m}$. This can be compared with those in previous studies with nonspectroscopic means. The timedomain charge measurement in Ref. [24] has demonstrated charge fractionalization of an incident wave packet, which yields the fractionalization ratio of $r=0.04$ in the amplitude, for the gate width $w \sim 1 \mu \mathrm{m}$. Based on the model shown in Sec. IV, this corresponds to $p \sim 4 r^{2} \sim 0.006$ in our parameter. Bolometric detection of heat transfer between counterpropagating channels in Ref. [25] have identified the interaction strength $|1-K|=0.1 \sim 0.25$, which corresponds to $p \sim 4\left(1-K^{-1}\right)^{2}=0.03 \sim 0.16$, depending on the gate voltage on the isolation gate of width $w \sim 0.2 \mu \mathrm{m}$. Since the fraction $p$ should increase with decreasing the separation of the counterpropagating channels, $p \sim 0.1$ in our case is reasonable for $w \sim 0.1 \mu \mathrm{m}$.

\section{B. Analogy with quantum quench}

The time dependence of the momentum spectrum (quantum quench) in Sec. IV can be related to the spatial dependence of the energy spectrum studied in Sec. III. The differences and similarities between them are summarized as follows.

Firstly, the energy (momentum) spectrum is given by the Fourier transform of the equal-position (equal-time) correlation function. Considering the noninteracting nondispersive plasmon modes with the constant velocities, the energy spectrum and the momentum spectrum should be related to each other $[29,36,40]$.

Secondly, the initial state that can be prepared by a PC shows a double-step distribution function [see the central inset of Fig. 1(b)], which is not in thermal equilibrium assumed in the quantum-quench simulation. Previous experiments for two copropagating channels [20], as well as our results in Sec. III B, resulted in a spectrum close to Fermi distribution function, which is similar to the spectra $f_{1}$ and $f_{2}$ in Fig. 9(b). This behavior can be reproduced in simulations of quantum quench starting from a double-step function in one channel [27] as well as from thermal equilibrium states at different temperatures [36]. This suggests that these different initial states do not play significant roles in the final state for the problem. Therefore, quantum quench starting from a Fermi distribution function is not a bad assumption to validate the appearance of binary spectrum in the system.

Thirdly, different from the quantum quench problem, the length of the interacting region in our experiment is finite. Thanks to the chiral nature of quantum-Hall edge channels, the plasmon transport is unidirectional particularly outside the interacting region. Nonequilibrium charge that left the interacting region never returns back. This prevents unwanted effects in the leads and dissipative ohmic contacts, and allows us to neglect the finite-length problem. Such unidirectional transport cannot be expected for conventional 1D wires connected to diffusive leads. In this way, our experiment can be regarded as a spatial analog of quantum quench.

\section{Conserved quantities during the transport}

Nonequilibrium transport in integer quantum Hall edge channels has been investigated for a few decades. When the scattering between the channels is well suppressed, the chemical potential of each channel is conserved during the transport, allowing the system to have independent charge distributions on the channels $[17,18]$. When the coupling to the environment, such as phonon bath, is negligible, the heat or the electron temperature of the system is conserved during the transport $[16,25,41]$. More intriguingly, when the interaction is not fully ergodic to cause thermal equilibration, the system may have infinite numbers of conserved quantities [42]. Our spectroscopic analysis has successfully revealed nonequilibrium electronic distribution with, at least, four conserved quantities (the binary spectrum parametrized by $T^{(\mathrm{H})}, T^{(\mathrm{L})}$, 

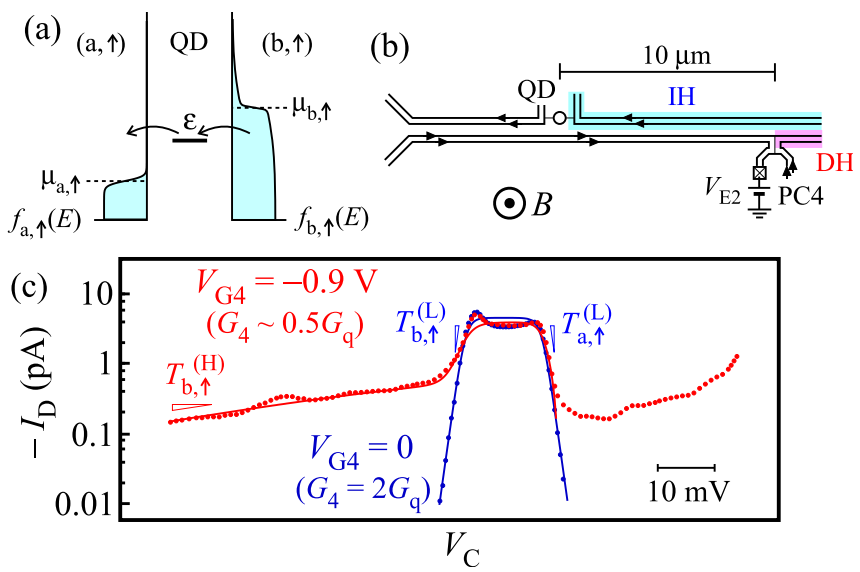

FIG. 10. Spectrum after traveling $10 \mu \mathrm{m}$. (a) The energy diagram at a negative bias $\mu_{\mathrm{a}, \uparrow}-\mu_{\mathrm{b}, \uparrow}=e V_{\mathrm{D}}=-80 \mu \mathrm{eV}$. (b) The location of $\mathrm{DH}$ and $\mathrm{IH}$ regions in the schematic channel layout. (c) Current spectrum of the QD measured with PC4 in the reversed magnetic field. Binary spectrum is seen on the left side of the peak only when the PC4 conductance is set in the tunneling regime $\left(G_{4} \simeq 0.5 G_{\mathrm{q}}\right)$.

$p$, as well as $\mu)$, which is greater than two $(\mu$ and $T)$ for trivial cases.

The TL theory suggests that the plasmons are noninteracting and thus should be conserved during the transport. This is supported by the fact that the nonequilibrium spectrum is sustained for a long distance. This is attractive for carrying much information as plasmon excitations rather than shortlived electronic excitations $[43,44]$.

\section{Possible relaxation mechanisms}

In previous reports, other energy transfer mechanisms such as phonons [45] or impurities [46] have been discussed to explain the charge and heat transfer. Such extrinsic effects with random processes are expected to result in a continuous thermalization into a trivial Fermi distribution. This contrasts with our binary spectrum independent of the propagation length (from $\sim 0$ to $10 \mu \mathrm{m}$ ), which implies that the extrinsic thermalization processes becomes relevant only at longer distances. This signifies the importance of Coulomb interaction in $1 \mathrm{D}$ systems prepared in the integer quantum Hall regime.

\section{SUMMARY}

We have investigated the energy spectrum of nonequilibrium states in quantum-Hall Tomonaga-Luttinger liquids. When nonequilibrium charge is injected from a PC, a nontrivial binary spectrum consisting of high- and low-temperature components appears in the $\mathrm{IH}$ region, while a seemingly thermalized spectrum appears in the DH region. The binary spectrum is sustained even after traveling 5-10 $\mu \mathrm{m}$, much longer than the length for electronic relaxation (about $0.1 \mu \mathrm{m}$ ), without showing significant thermalization. This can be compared with the simulation of quantum quench problem, which also suggest the emergence of an approximate binary spectrum as a nonequilibrium steady state. The long-lived binary spectrum implies that the system is well described by noninteracting plasmons, which suggests that low-energy excitations of edge channels in the integer quantum Hall regime can be well understood as a TL liquid.

\section{ACKNOWLEDGMENTS}

We would like to thank Tsuneya Ando, Norio Kawakami, Tomohiro Sasamoto, and Masahito Ueda for fruitful discussions. This work was supported by Japan Society for the Promotion of Science (JSPS) KAKENHI (Grants No. 21000004, No. 12J09291, No. 24009291, No. 26247051, and No. 15H05854), International Research Center for Nanoscience and Quantum Physics at Tokyo Institute of Technology, and Nanotechnology Platform Program of the Ministry of Education,Culture,Sports,Science and Technology, Japan.

\section{APPENDIX A: FITTING PROCEDURE}

The fitting curves in Figs. 3-5 are obtained by using Eq. (1) with a constant current level $I_{0}$ as a free parameter. The dot energy $\varepsilon=\alpha\left(V_{\mathrm{C}}-V_{\mathrm{C}, 0}\right)$ is converted from the gate voltage $V_{\mathrm{C}}$ with a factor $\alpha \simeq 0.018 e$ from an offset $V_{\mathrm{C}, 0}$. For the spectrum in the DH region in Fig. 3(b), the Fermi distribution functions [Eq. (2)] with free parameters $T_{\mathrm{a} / \mathrm{b}, \uparrow}$ and $\mu_{\mathrm{a} / \mathrm{b}, \uparrow}$ are used. When binary spectrum is found in the IH regions (Figs. 4 and 5), the binary spectra of the form Eq. (3) with free parameters $p_{\mathrm{a} / \mathrm{b}, \uparrow}, T_{\mathrm{a} / \mathrm{b}, \uparrow}^{(\mathrm{L},}, T_{\mathrm{a} / \mathrm{b}, \uparrow}^{(\mathrm{H})}$, and $\mu_{\mathrm{a} / \mathrm{b}, \uparrow}$ are used. We fitted $I_{\text {fit }}(\varepsilon)$ to the measured data $I_{\text {meas }}(\varepsilon)$ by minimizing the residual error $\int\left[\log I_{\text {meas }}(\varepsilon)-\log I_{\text {fit }}(\varepsilon)\right]^{2} d \varepsilon$ evaluated in the logarithmic scale to focus on the low-current profile. In order to obtain reliable fitting, data with current level smaller than 10-20 fA were discarded. The hot minority spectrum with wide current level ranging from 10-200 fA is analyzed. Since small fitting error always exists near the transition between the hot and cold spectrum, the fitting involves ambiguity as shown by error bars in Figs. 6(c) and 8(c). When $T^{(\mathrm{H})}$ is high, the spectrum is extended over a wide energy range greater than the typical energy spacing of $200 \mu \mathrm{eV}$. We neglected any effects from the excited states and energy-dependent tunneling rate, which might give additional error in the estimate of $T^{(\mathrm{H})}$.

\section{APPENDIX B: BINARY SPECTRUM AFTER TRAVELING $10 \mu \mathrm{m}$}

The spectrum measured at the farthest position downstream in the IH region is obtained with PC4 in the reversed field at $B=-5.9$ T. As shown in Fig. 10(b), QD is located $10 \mu \mathrm{m}$ downstream in the IH region from PC4. As shown in Fig. 10(c), a binary spectrum emerges on the left side of the peak only when a large excitation voltage $\left(V_{\mathrm{E} 2}=2 \mathrm{mV}\right)$ is applied across PC4 in the tunneling regime $\left(G_{4} \simeq 0.5 G_{\mathrm{q}}\right.$ at $\left.V_{\mathrm{G} 4}=-0.9 \mathrm{~V}\right)$. The profile can be fitted with the binary spectrum of hot minority carriers $\left(k_{\mathrm{B}} T_{\mathrm{b}, \uparrow}^{(\mathrm{H})} \simeq 350 \mu \mathrm{eV}\right.$ and $\left.p \simeq 0.25\right)$ and cold majority carriers $\left(k_{\mathrm{B}} T_{\mathrm{b}, \uparrow}^{(\mathrm{L})} \simeq 15 \mu \mathrm{eV}\right)$ as shown by the thin solid line. The large current on the right of the peak is associated with a similar binary spectrum detected with the next Coulomb blockade peak. Note that, for this experiment, negative bias $V_{\mathrm{D}}=-80 \mu \mathrm{V}$ is applied to the channel $(\mathrm{b}, \sigma)$ to make the transport go from the right to the left as shown in Fig. 10(a). Therefore, the spectrum of (b, $\uparrow$ ) appears on the left side of the peak, which is different from the data set in Fig. 4. 
[1] J. Gemmer, M. Michel, and G. Mahler, Quantum Thermodynamics: Emergence of Thermodynamic Behavior Within Composite Quantum Systems, Lecture Notes in Physics Vol. 784 (Springer, Berlin Heidelberg, 2006).

[2] A. Polkovnikov, K. Sengupta, A. Silva, and M. Vengalattore, Rev. Mod. Phys. 83, 863 (2011).

[3] T. Kinoshita, T. Wenger, and D. S. Weiss, Nature (London) 440, 900 (2006).

[4] M. Gring, M. Kuhnert, T. Langen, T. Kitagawa, B. Rauer, M. Schreitl, I. Mazets, D. A. Smith, E. Demler, and J. Schmiedmayer, Science 337, 1318 (2012).

[5] Z. F. Ezawa, Quantum Hall Effects: Field Theoretical Approach and Related Topics (World Scientific, Singapore, 2008).

[6] A. M. Chang, Rev. Mod. Phys. 75, 1449 (2003).

[7] H. Steinberg, G. Barak, A. Yacoby, L. N. Pfeiffer, K. W. West, B. I. Halperin, and K. Le Hur, Nat. Phys. 4, 116 (2008).

[8] G. Barak, H. Steinberg, L. N. Pfeiffer, K. W. West, L. Glazman, F. von Oppen, and A. Yacoby, Nat. Phys. 6, 489 (2010).

[9] C. Blumenstein, J. Schafer, S. Mietke, S. Meyer, A. Dollinger, M. Lochner, X. Y. Cui, L. Patthey, R. Matzdorf, and R. Claessen, Nat. Phys. 7, 776 (2011).

[10] S. Tomonaga, Prog. Theor. Phys. 5, 544 (1950).

[11] J. M. Luttinger, J. Math. Phys. 4, 1154 (1963).

[12] T. Giamarchi, Quantum Physics in One Dimension (Oxford University Press, Oxford, 2004).

[13] M. A. Cazalilla, Phys. Rev. Lett. 97, 156403 (2006).

[14] A. Iucci and M. A. Cazalilla, Phys. Rev. A 80, 063619 (2009).

[15] D. M. Kennes, C. Klöckner, and V. Meden, Phys. Rev. Lett. 113, 116401 (2014).

[16] S. Jezouin, F. D. Parmentier, A. Anthore, U. Gennser, A. Cavanna, Y. Jin, and F. Pierre, Science 342, 601 (2013).

[17] M. Büttiker, Phys. Rev. B 38, 9375(R) (1988).

[18] B. J. van Wees, E. M. M. Willems, L. P. Kouwenhoven, C. J. P. M. Harmans, J. G. Williamson, C. T. Foxon, and J. J. Harris, Phys. Rev. B 39, 8066 (1989).

[19] C. Altimiras, H. le Sueur, U. Gennser, A. Cavanna, D. Mailly, and F. Pierre, Nat. Phys. 6, 34 (2010).

[20] H. le Sueur, C. Altimiras, U. Gennser, A. Cavanna, D. Mailly, and F. Pierre, Phys. Rev. Lett. 105, 056803 (2010).

[21] E. Bocquillon, V. Freulon, J. M. Berroir, P. Degiovanni, B. Plaçais, A. Cavanna, Y. Jin, and G. Fève, Nat. Commun. 4, 1839 (2013).

[22] H. Inoue, A. Grivnin, N. Ofek, I. Neder, M. Heiblum, V. Umansky, and D. Mahalu, Phys. Rev. Lett. 112, 166801 (2014).

[23] V. Freulon, A. Marguerite, J. M. Berroir, B. Placais, A. Cavanna, Y. Jin, and G. Feve, Nat. Commun. 6, 6854 (2015).
[24] H. Kamata, N. Kumada, M. Hashisaka, K. Muraki, and T. Fujisawa, Nat. Nanotechnol. 9, 177 (2014).

[25] M. G. Prokudina, S. Ludwig, V. Pellegrini, L. Sorba, G. Biasiol, and V. S. Khrapai, Phys. Rev. Lett. 112, 216402 (2014).

[26] P. Degiovanni, Ch. Grenier, G. Fève, C. Altimiras, H. le Sueur, and F. Pierre, Phys. Rev. B 81, 121302 (2010).

[27] D. L. Kovrizhin and J. T. Chalker, Phys. Rev. Lett. 109, 106403 (2012).

[28] D. B. Gutman, Y. Gefen, and A. D. Mirlin, Phys. Rev. Lett. 101, 126802 (2008).

[29] D. B. Gutman, Y. Gefen, and A. D. Mirlin, Phys. Rev. B 80, 045106 (2009).

[30] E. Berg, Y. Oreg, E.-A. Kim, and F. von Oppen, Phys. Rev. Lett. 102, 236402 (2009).

[31] M. Hashisaka, H. Kamata, N. Kumada, K. Washio, R. Murata, K. Muraki, and T. Fujisawa, Phys. Rev. B 88, 235409 (2013).

[32] I. Safi and H. J. Schulz, Phys. Rev. B 52, R17040 (1995).

[33] K. I. Imura, K. V. Pham, P. Lederer, and F. Piechon, Phys. Rev. B 66, 035313 (2002).

[34] H. Kamata, T. Ota, K. Muraki, and T. Fujisawa, Phys. Rev. B 81, 085329 (2010).

[35] N. Kumada, H. Kamata, and T. Fujisawa, Phys. Rev. B 84, 045314 (2011).

[36] D. L. Kovrizhin and J. T. Chalker, Phys. Rev. B 84, 085105 (2011).

[37] I. P. Levkivskyi and E. V. Sukhorukov, Phys. Rev. B 85, 075309 (2012).

[38] E. Onac, F. Balestro, L. H. van Beveren, U. Hartmann, Y. V. Nazarov, and L. P. Kouwenhoven, Phys. Rev. Lett. 96, 176601 (2006).

[39] J. von Delft and H. Schoeller, Ann. Phys. 7, 225 (1998).

[40] P. Jurcevic, B. P. Lanyon, P. Hauke, C. Hempel, P. Zoller, R. Blatt, and C. F. Roos, Nature (London) 511, 202 (2014).

[41] G. Granger, J. P. Eisenstein, and J. L. Reno, Phys. Rev. Lett. 102, 086803 (2009).

[42] J. Berges, S. Borsányi, and C. Wetterich, Phys. Rev. Lett. 93, 142002 (2004).

[43] Y. Ji, Y. Chung, D. Sprinzak, M. Heiblum, D. Mahalu, and H. Shtrikman, Nature (London) 422, 415 (2003).

[44] I. P. Levkivskyi and E. V. Sukhorukov, Phys. Rev. B 78, 045322 (2008).

[45] M. G. Prokudina, V. S. Khrapai, S. Ludwig, J. P. Kotthaus, H. P. Tranitz, and W. Wegscheider, Phys. Rev. B 82, 201310(R) (2010).

[46] A. M. Lunde, S. E. Nigg, and M. Büttiker, Phys. Rev. B 81, 041311 (2010). 